Center for Outcomes Research and Evaluation, Yale New Haven Hospital, New Haven, Connecticut, USA

2 Division of Cardiac Surgery, Yale School of Medicine, New Haven, Connecticut, USA

3 Harvard Medical School, Boston, Massachusetts, USA

4 Department of Internal Medicine, Yale School of Medicine, New Haven, Connecticut, USA

5 Section of Cardiovascular Medicine, Department of Internal Medicine, Yale School of Medicine and the Department of Health Policy and Management, Yale School of Public Health, New Haven, Connecticut, USA

Cite this as: BMJ2022;376:0525 http://dx.doi.org/10.1136/bmj.0525 Published: 28 February 2022

\section{Sensible regulation and clinical implementation of clinical decision support software as a medical device}

\author{
Makoto Mori, ${ }^{1,2}$ Robert Jarrin, ${ }^{1}$ Yuan Lu, ${ }^{1}$ Kushal Kadakia, ${ }^{3}$ Chenxi Huang, ${ }^{1}$ Joseph S. Ross, ${ }^{1,4}$ \\ Harlan M. Krumholz', 5
}

Recently published research determined that the Epic Systems Corporation Sepsis Model-a widely implemented electronic health record (EHR) based software to identify patients with sepsis-performed poorly at identifying patients at risk for sepsis compared with standard workflows, despite generating added burden for clinicians. ${ }^{1}$ While the company had internally validated the model in 2015 , limited information was made public about the algorithm's performance because it-like many other clinical decision support tools-did not require clearance from the United States Food and Drug Administration (FDA) before being used in patient care.

The FDA defines clinical decision support tools as a category of "Software as a Medical Device (SaMD) products." While the promise of clinical decision support tools is to help clinicians make faster and more accurate decisions, the peril is that for many there are no regulatory requirements for testing or validation before implementation in the clinical care setting.

In the traditional framework of device regulation and distribution, manufacturers typically must follow general controls and obtain FDA approval or clearance before marketing or updating devices. ${ }^{2}$ This applies to whether a device is primarily hardware, software, or both. However, regulatory oversight is not consistently required for many Software as a Medical Device products, such as web based or EHR based clinical decision support tools that are made available for use by clinicians who are unaware that it is their responsibility to independently assess their validity and performance. A developer could publish a faulty algorithm, and the onus is on the practitioner to independently review and evaluate the basis of the recommendation. Thus, whether the clinical decision support tool is regulated has great importance.

In 2019, the FDA issued the "Clinical Decision Support Software” Draft Guidance document. ${ }^{3}$ It stated that if the intended user of the clinical decision support is a healthcare professional who can "independently review the basis for the recommendation" the software would be considered non-device clinical decision support, and no regulatory oversight would be required. The field has continued to evolve rapidly since the draft guidance's publication, and the development and adoption of new clinical decision support tools raises additional questions about evidence quality and real world safety monitoring. Meanwhile, stakeholders eagerly anticipate the FDA's publication of a final clinical decision support guidance document, seeking further clarity about which clinical decision support will be regulated and how regulatory oversight will be implemented across the product life cycle. ${ }^{4}$

The problem with exempting FDA review based on the clinician's ability to examine and understand the clinical decision support tool is that most clinicians lack the expertise and time to navigate the original development and validation literature to independently interpret or review the basis for the clinical decision support output. The increasing technical complexity of such models, including those driven by varying degrees of assistive, augmentative, and autonomous artificial intelligence (AI) applications (e.g., expert systems, machine learning, algorithm-based services), precludes most physicians from making such an assessment. Moreover, the exact analytic and data methods employed may not be publicly available, or at least easily accessible. And even if doctors have the skills and the access to the data, placing the decision on individual clinicians at the point of practice (as opposed to in more formal, deliberative settings by consensus-based review) is perhaps not the best way to provide oversight of these clinical decision support tools.

While the degree of regulatory oversight must be reasonable and balanced to allow for innovation and iterative refinement, it is unreasonable to expect clinicians to evaluate the technical nuance of each clinical decision support tool and decide how best to use the output. Moreover, many of these models are developed on homogenous patient populations, lacking representation of minority patients or perhaps patients with certain diseases. ${ }^{5}$ Furthermore, if the clinical decision support algorithms are designed to be continuous learning models, then there needs to be clarity about standards and processes for ongoing good machine learning practices and labelling, as frequent updates to models can cause a drift in clinical decision support performance when applied to an individual clinician's practice. ${ }^{4}$

While clarifying premarket standards for clinical decision support use is a helpful first step at mitigating potential risks, it is possible that issues with safety and performance with software-just like other medical products like drugs and devices-may not be fully apparent until they are implemented in real-world settings. Consequently, there is a need to develop post-market surveillance mechanisms for clinical decision support tools, which in the status quo lack reliable methodologies for identifying safety signals. Adverse events related to regulated clinical decision support tools should be reported through the FDA's adverse event surveillance system, but what constitutes an adverse event for clinical decision 
support tools is unclear and users may lack knowledge about how to report it. Moreover, with clinical decision support, adverse events may also relate to performance issues, which can only be detected by systematic study of the clinical decision support.

Congress, through the 21st Century Cures Act, introduced the language of "independent review" that underpins the FDA's draft clinical decision support guidance. ${ }^{6}$ Consequently, the agency may have limited means to impose a different standard on clinical decision support. However, as Congress considers Cures 2.0, it may seek to remedy this regulatory approach that has opened the marketplace to tools that have not undergone rigorous testing. Ideally, the current clinical decision support Draft Guidance may be strengthened by accounting for the following seven suggestions:

1. A risk based strategy, with mandated regulation where the clinical decision support has important clinical relevance and errors could jeopardize patient outcomes with exceptions only for when the clinical decision support logic is obvious to people without any training in statistics or epidemiology because it is a simple rule-based approach.

2. A venue-based strategy, with mandated regulation for all clinical decision support instruments that are integrated in electronic health records and available at the point of care.

3. A commitment to public access to the methods employed for clinical decision support that has any clinical relevance-and available evidence about the performance characteristics of the clinical decision support.

4. A visible and user-friendly mechanism for the reporting of adverse events associated with clinical decision support.

5. A cadence of re-evaluation and modification throughout the product life cycle for regulated clinical decision support tools.

6. A post-market evaluation strategy for regulated clinical decision support tools so that there is a systematic evaluation of performance in real-world settings.

7. Clear messaging, particularly oriented to clinicians, for all unregulated clinical decision support tools, in clear language signalling that the tool has not gone through regulatory oversight. If the clinical decision support tool has not been tested, then that information should be clearly posted.

Clinical decision support tools have great promise to improve medical practice and patient outcomes. However, as health systems' experience demonstrates, the use of clinical decision support in the current regulatory environment carries the risk of unintended adverse consequences. The net benefit of clinical decision support tools will depend on wise regulation-and clearing signalling to the public when no regulatory oversight has occurred.

Competing interests: In the past three years, Harlan Krumholz received expenses and/or personal fees from UnitedHealth, Element Science, Aetna, Reality Laboratories, Tesseract/4Catalyst, the Siegfried and Jensen Law Firm, Arnold and Porter Law Firm, Martin/Baughman Law Firm, and F-Prime. He is a co-founder of Refactor Health and HugoHealth, and is associated with contracts, through Yale New Haven Hospital, from the Centers for Medicare and Medicaid Services and through Yale University from Johnson and Johnson.

BMJ has a decision support tool called Best Practice

Provenance and peer review: not commissioned, not externally peer reviewed

Wong A, Otles E, Donnelly JP, etal. External validation of a widely implemented proprietary sepsis prediction model in hospitalized patients. JAMA Intern Med 2021;181:1065-70.

doi: 10.1001/jamainternmed.2021.2626. pmid: 34152373
2 U.S. Food \& Drug Administration. How to study and market your device. U.S. Food and Drug Administration. Published December 14, 2020. Accessed January 24, 2022. https://www.fda.gov/medical-devices/device-advice-comprehensive-regulatory-assistance/howstudy-and-market-your-device

3 U.S. Food \& Drug Administration. Clinical decision support software. U.S. Food and Drug Administration. Published May 6, 2020. Accessed January 19, 2022. https://www.fda.gov/regulatory-information/search-fda-guidance-documents/clinical-decision-support-software

4 Hwang TJ, Kesselheim AS, Vokinger KN. Lifecycle regulation of artificial intelligence- and machine learning-based software devices in medicine. JAMA 2019;322:2285-6. doi: 10.1001/jama.2019.16842. pmid: 31755907

5 Pfohl SR, Foryciarz A, Shah NH. An empirical characterization of fair machine learning for clinical risk prediction. J Biomed Inform2021;113:103621. doi: 10.1016/j.jbi.2020.103621. pmid: 33220494

6 U.S. Food \& Drug Administration. Software as a Medical Device (SAMD): Clinical evaluation guidance for industry and Food and Drug Administration Staff. Published online 2017. Accessed January 22, 2022. https://www.fda.gov/regulatory-information/search-fda-guidance-documents/software-medical-device-samd-clinical-evaluation. 